\title{
Convergence Rate of Numerical Solutions for Nonlinear Stochastic Pantograph Equations with Markovian Switching and Jumps
}

\author{
Zhenyu Lu, ${ }^{1}$ Tingya Yang, ${ }^{2}$ Yanhan $\mathrm{Hu}^{3}$ and Junhao $\mathrm{Hu}^{3}$ \\ ${ }^{1}$ College of Electrical and Information Engineering, Nanjing University of Information Science \& Technology, \\ Nanjing 210044, China \\ ${ }^{2}$ Jiangsu Meteorological Observatory, Nanjing 210008, China \\ ${ }^{3}$ College of Mathematics and Statistics, South-Central University for Nationalities, Wuhan 430074, China
}

Correspondence should be addressed to Junhao Hu; junhaomath@163.com

Received 8 April 2013; Accepted 5 June 2013

Academic Editor: Zidong Wang

Copyright ( 2013 Zhenyu Lu et al. This is an open access article distributed under the Creative Commons Attribution License, which permits unrestricted use, distribution, and reproduction in any medium, provided the original work is properly cited.

\begin{abstract}
The sufficient conditions of existence and uniqueness of the solutions for nonlinear stochastic pantograph equations with Markovian switching and jumps are given. It is proved that Euler-Maruyama scheme for nonlinear stochastic pantograph equations with Markovian switching and Brownian motion is of convergence with strong order $1 / 2$. For nonlinear stochastic pantograph equations with Markovian switching and pure jumps, it is best to use the mean-square convergence, and the order of mean-square convergence is close to $1 / 2$.
\end{abstract}

\section{Introduction}

Stochastic modelling has been used with great success in a variety of application areas, including control theory, biology, epidemiology, mechanic, and neural networks, economics, and finance [1-5]. In general, stochastic different equations do not have explicit solutions. Therefore, approximate schemes for stochastic differential equations with Markovian switching and Poisson jumps have been investigated by many authors $[3,6,7]$. The convergence results of numerical solutions of stochastic differential equations with Markovian switching and Poisson jumps under the Lipschitz condition and the linear growth condition are obtained by using EulerMaruyama scheme or semi-implicit Euler scheme. However, recently, more and more convergence results have been given under weaker conditions than the Lipschitz condition and the linear growth condition. Gyöngy and Rásonyi [8] revealed the convergence rate of Euler approximations for stochastic differential equations whose diffusion coefficient is not Lipschitz but only $(1 / 2+\alpha)$-Hölder continuous for some $\alpha>0$. Mao et al. [9] discussed $L^{1}$ and $L^{2}$-convergence of the Euler-Maruyama scheme for stochastic differential equations with Markovian switching under non-Lipschitz coefficients. Wu et al. [10] proved existence of the nonnegative and the strong convergence of the Euler-Maruyama Scheme for the Cox-Ingersoll-Ross model with delay whose diffusion coefficient is nonlinear and non-Lipschitz continuous. Bao and Yuan [11] studied the convergence rate for stochastic differential delay equations whose coefficients may be highly nonlinear with respect to the delay variable.

So far, the research of the numerical solutions for stochastic pantograph equations has just begun [12-15]. Fan et al. [12] gave the strong convergence for stochastic pantograph equations under the Lipschitz condition and the linear growth condition. Ronghua et al. [14] proved that the Euler approximation solution converges to the analytic solution in probability under weaker conditions, but the convergence rate has not been given.

In this paper, we will study the convergence rate for nonlinear stochastic pantograph equations with Markovian switching and Poisson jump under weaker conditions than the Lipschitz condition and the linear growth condition. The rest of the paper is organized as follows. In Section 2, we will give the existence and uniqueness of the analytic solutions 
for Markovian switching and Brownian motion case and also reveal that the convergence order of Euler-Maruyama scheme is $1 / 2$. In Section 3, we show that it is best to use the meansquare convergence for Markovian switching and the pure jump case and that the rate of mean-square convergence is close to $1 / 2$.

\section{Convergence Rate for Markovian Switching and Brownian Motion Case}

Let $(\Omega, \mathscr{F}, \mathbb{P})$ be a complete probability space with a filtration $\left\{\mathscr{F}_{t}\right\}_{t \geq 0}$ satisfying the usual conditions. Let $W(t)$ be an $m$-dimensional Brownian motion defined on the probability space adapted to the filtration. For integer $n>$ 0 , let $\left(\mathbb{R}^{n},\langle\cdot, \cdot\rangle,|\cdot|\right)$ be the Euclidean space and $\|A\|:=$ $\sqrt{\operatorname{trace}\left(A^{*} A\right)}$ the Hilbert-Schmidt norm for a matrix $A$, where $A^{*}$ is its transpose. Throughout this paper, $C>0$ denotes a generic constant whose values may change from lines to lines.

Let $r(t), t \geq 0$ be a right-continuous Markov chain on the probability space taking values in a finite state space $S=$ $\{1,2, \ldots, N\}$ with the generator $\Gamma=\left(\gamma_{i j}\right)_{N \times N}$ given by

$$
\mathbb{P}\{r(t+\delta)=j \mid r(t)=i\}= \begin{cases}\gamma_{i j} \delta+o(\delta) & \text { if } i \neq j \\ 1+r_{i j} \delta+o(\delta) & \text { if } i=j\end{cases}
$$

where $\delta>0$. Here $\gamma_{i j}>0$ is the transition rate from $i$ to $j$ if $i \neq j$ while

$$
\gamma_{i i}=-\sum_{j \neq i} \gamma_{i j}
$$

We assume that the Markov chain $r(\cdot)$ is independent of the Brownian motion $W(\cdot)$. It is well known that almost every sample path of $r(\cdot)$ is a right continuous step function with finite number of sample jumps in any finite subinterval of $\mathbb{R}_{+}:=[0,+\infty)$.

For fixed $T>0$, we consider the stochastic pantograph equation with Markovian switching of the form

$$
\begin{aligned}
\mathrm{d} X(t)= & b(X(t), X(q t), r(t)) \mathrm{d} t \\
& +\sigma(X(t), X(q t), r(t)) \mathrm{d} W(t), \quad t \in\left[t_{0}, T\right],
\end{aligned}
$$

with initial data $X(\theta)=\xi(\theta), r(\theta)=r_{0}, \theta \in\left[q t_{0}, t_{0}\right], 0<$ $t_{0}, 0<q<1 . r(t)$ is a Markov chain. On the time interval $\left[t_{0}, T\right]$, let $q<\epsilon<\min \{1,((T+1) / T) q\}$, and we define the partition

$$
\begin{aligned}
0 & <t_{0}<\frac{t_{0}}{q} \epsilon<\frac{t_{0}}{q^{2}} \epsilon^{2}<\frac{t_{0}}{q^{3}} \epsilon^{3} \\
& <\cdots<\frac{t_{0}}{q^{n-1}} \epsilon^{n-1}<\frac{t_{0}}{q^{n}} \epsilon^{n}, \quad n=\left[\log _{\epsilon / q} \frac{T}{t_{0}}\right]+1, \\
0 & <\Delta_{i}=\frac{t_{0}}{q^{i}} \epsilon^{i}-\frac{t_{0}}{q^{i-1}} \epsilon^{i-1}=\frac{t_{0}}{q^{i}} \epsilon^{i}\left(1-\frac{q}{\epsilon}\right) \\
& <\frac{\epsilon}{q} T\left(1-\frac{q}{\epsilon}\right)=\left(\frac{\epsilon}{q}-1\right) T<1 .
\end{aligned}
$$

The integral version of (3) is given by the following:

$$
\begin{aligned}
X(t)= & \xi(\theta)+\int_{t_{0}}^{t} b(X(s), X(q s), r(s)) \mathrm{d} s \\
& +\int_{t_{0}}^{t} \sigma(X(s), X(q s), r(s)) \mathrm{d} W(s) .
\end{aligned}
$$

To guarantee the existence and uniqueness of the solutions of (3) we introduce the following conditions:

(A1) $b: \mathbb{R}^{n} \times \mathbb{R}^{n} \times S \rightarrow \mathbb{R}^{n}$ and there exists $L_{1}>0$ such that

$$
\begin{aligned}
& \left|b\left(x_{1}, y_{1}, j\right)-b\left(x_{2}, y_{2}, j\right)\right| \\
& \quad \leq L_{1}\left|x_{1}-x_{2}\right|+V_{1}\left(y_{1}, y_{2}\right)\left|y_{1}-y_{2}\right|
\end{aligned}
$$

for $x_{i}, y_{i} \in \mathbb{R}^{n}, i=1,2, j \in S$;

(A2) $\sigma: \mathbb{R}^{n} \times \mathbb{R}^{n} \times S \rightarrow \mathbb{R}^{n \times m}$ and there exists $L_{2}>0$ such that

$$
\begin{aligned}
& \qquad \begin{array}{l}
\left\|\sigma\left(x_{1}, y_{1}, j\right)-\sigma\left(x_{2}, y_{2}, j\right)\right\| \\
\quad \leq L_{2}\left|x_{1}-x_{2}\right|+V_{2}\left(y_{1}, y_{2}\right)\left|y_{1}-y_{2}\right|
\end{array} \\
& \text { for } x_{i}, y_{i} \in \mathbb{R}^{n}, i=1,2, j \in S,
\end{aligned}
$$

where $V_{i}: \mathbb{R}^{n} \times \mathbb{R}^{n} \rightarrow \mathbb{R}_{+}$such that

$$
V_{i}(x, y) \leq K_{i}\left(1+|x|^{q_{i}}+|y|^{q_{i}}\right), \quad i=1,2
$$

for some $K_{i}>0, q_{i} \geq 1$ and arbitrary $x, y \in \mathbb{R}^{n}$.

Remark 1. From (A1)-(A2), we know that the coefficients of (3) are much weaker than those of the Lipschitz condition and the linear growth condition. In many examples, $b$ and $\sigma$ do not satisfy the Lipschitz condition or the linear growth condition but can be covered by (A1)-(A2).

Lemma 2. Assume that (A1) and (A2) hold. Then, for any initial data $\xi \in C_{\mathscr{F}_{0}}^{b}\left(\left[q t_{0}, t_{0}\right] ; \mathbb{R}^{n}\right)$ and $r(0)=r_{0} \in S, X(t)$ is a unique global strong solution of (3). Moreover, for any $p \geq 2$ there exists $C>0$ such that

$$
\mathbb{E}\left(\sup _{t_{0} \leq t \leq T}|X(t)|^{p}\right) \leq C .
$$

Proof. From (A1) and (A2), $b$ and $\sigma$ are locally Lipschitzian. So, (3) has a unique local solution [3]. In order to verify that (3) has a unique global solution on time interval $\left[t_{0}, T\right]$, it is sufficient to show that

$$
\mathbb{E}\left(\sup _{t_{0} \leq t \leq T}|X(t)|^{p}\right) \leq C, \quad p \geq 2 .
$$

From (A1), (A2), and (8), we can obtain

$$
\begin{gathered}
|b(x, y, i)| \leq C\left(1+|x|+|y|+|y|^{q_{1}+1}\right), \quad x, y \in \mathbb{R}^{n}, \\
\|\sigma(x, y, i)\| \leq C\left(1+|x|+|y|+|y|^{q_{2}+1}\right), \quad x, y \in \mathbb{R}^{n} .
\end{gathered}
$$


Substituting (11) and (12) into (5) and by the Hölder inequality and the Burkhold-Davis-Gundy inequality, we have that for any $p \geq 2$ and $t \in\left[t_{0}, T\right]$

$$
\begin{aligned}
& \mathbb{E}\left(\sup _{t_{0} \leq s \leq t}|X(s)|^{p}\right) \\
& \leq 3^{p-1}\left\{|\xi(\theta)|^{p}\right. \\
& +\mathbb{E}\left(\sup _{t_{0} \leq s \leq t}\left|\int_{t_{0}}^{s} b(X(\alpha), X(q \alpha), r(\alpha)) \mathrm{d} \alpha\right|^{p}\right) \\
& \left.+\mathbb{E}\left(\sup _{t_{0} \leq s \leq t}\left|\int_{t_{0}}^{s} \sigma(X(\alpha), X(q \alpha), r(\alpha)) \mathrm{d} W(\alpha)\right|^{p}\right)\right\} \\
& \leq C\left\{1+\mathbb{E} \int_{t_{0}}^{t}\left(|b(X(s), X(q s), r(s))|^{p}\right.\right. \\
& \leq C\left\{1+\mathbb{E} \int_{t_{0}}^{t}|X(s)|^{p} \mathrm{~d} s\right. \\
& \left.+\mathbb{E} \int_{t_{0}}^{t}\left(|X(q s)|^{p\left(q_{1}+1\right)}+|X(q s)|^{p\left(q_{2}+1\right)}\right) \mathrm{d} s\right\} .
\end{aligned}
$$

Let $\beta:=\left(q_{1}+1\right) \vee\left(q_{2}+1\right)$; then

$$
\begin{aligned}
& \mathbb{E}\left(\sup _{t_{0} \leq s \leq t}|X(s)|^{p}\right) \\
& \quad \leq C\left\{1+\mathbb{E} \int_{t_{0}}^{t}|X(s)|^{p} \mathrm{~d} s+\mathbb{E} \int_{t_{0}}^{t}|X(q s)|^{p \beta} \mathrm{d} s\right\} .
\end{aligned}
$$

By virtue of the Gronwall inequality, we get

$$
\mathbb{E}\left(\sup _{t_{0} \leq s \leq t}|X(s)|^{p}\right) \leq C\left\{1+\mathbb{E} \int_{t_{0}}^{t}|X(q s)|^{p \beta} \mathrm{d} s\right\} .
$$

Let

$$
\begin{array}{r}
p_{i}:=\left(\left[\log _{\epsilon / q} \frac{T}{t_{0}}\right]+2-i\right) p \beta^{\left[\log _{\epsilon / q}\left(T / t_{0}\right)\right]+1-i}, \\
i=1,2, \ldots,\left[\log _{\epsilon / q} \frac{T}{t_{0}}\right]+1,
\end{array}
$$

where $[a]$ denotes the integer part of real number $a$; thus, for $\beta \geq 1$ and $p \geq 2$, we have

$$
\begin{array}{r}
p_{i+1} \beta<p_{i}, \quad p_{\left[\log _{\epsilon / q}\left(T / t_{0}\right)\right]+1}=p \\
i=1,2, \ldots,\left[\log _{\epsilon / q} \frac{T}{t_{0}}\right] .
\end{array}
$$

Together with $\xi \in C_{\mathscr{F}_{0}}^{b}\left(\left[q t_{0}, t_{0}\right] ; \mathbb{R}^{n}\right)$ and $\epsilon<1$, we obtain that

$$
\begin{aligned}
& \mathbb{E}\left(\sup _{t_{0} \leq s \leq\left(t_{0} / q\right) \epsilon}|X(s)|^{p_{1}}\right) \\
& \quad \leq C\left\{1+\mathbb{E} \int_{t_{0}}^{\left(t_{0} / q\right) \epsilon}|X(q s)|^{p_{1} \beta} \mathrm{d} s\right\} \\
& \quad \leq C\left\{1+\mathbb{E} \int_{q t_{0}}^{t_{0}}|X(s)|^{p_{1} \beta} \mathrm{d} s\right\} \\
& \leq C .
\end{aligned}
$$

In the similar way, combining (15) with the Hölder inequality further leads to

$$
\begin{aligned}
& \mathbb{E}\left(\sup _{t_{0} \leq s \leq\left(t_{0} / q^{2}\right) \epsilon^{2}}|X(s)|^{p_{2}}\right) \\
& \quad \leq C\left\{1+\mathbb{E} \int_{t_{0}}^{\left(t_{0} / q^{2}\right) \epsilon^{2}}|X(q s)|^{p_{2} \beta} \mathrm{d} s\right\} \\
& \quad \leq C\left\{1+\int_{t_{0}}^{\left(t_{0} / q\right) \epsilon}\left(\mathbb{E}|X(s)|^{p_{1}}\right)^{p_{2} \beta / p_{1}} \mathrm{~d} s\right\} \\
& \leq C .
\end{aligned}
$$

Repeating the previous procedures we then get (9). So the existence and uniqueness have been proved.

In the following, we define the Euler-Maruyama based computational method. The method makes use of the following lemma.

Lemma 3. Given $\Delta>0$, then $\{r(k \Delta), k=0,1,2, \ldots\}$ is a discrete Markov chain with the one-step transition probability matrix

$$
P(\Delta)=\left(P_{i, j}(\Delta)\right)_{N \times N}=e^{\Delta \Gamma}
$$

Given a fixed step size $\Delta>0$ and the one-step transition probability matrix $P(\Delta)$ in (20), the discrete Markov chain $\{r(k \Delta), k=0,1,2, \ldots\}$ can be simulated as follows: let $r(0)=i_{0}$, and compute a pseudorandom number $\xi_{1}$ from the uniform $(0,1)$ distribution.

Define

$$
r(\Delta)= \begin{cases}i, & i \in S-\{N\} \text { such that } \sum_{j=1}^{i-1} P_{r(0), j}(\Delta) \leq \xi_{1} \\ & <\sum_{j=1}^{i} P_{r(0), j}(\Delta), \\ N, & \sum_{j=1}^{N-1} P_{r(0), j}(\Delta) \leq \xi_{1},\end{cases}
$$


where we set $\sum_{j=1}^{0} P_{r(0), j}(\Delta)=0$ as usual. Having computed $r(0), r(\Delta), \ldots, r(k \Delta)$, we can compute $r((k+1) \Delta)$ by drawing a uniform $(0,1)$ pseudo-random number $\xi_{k+1}$ and setting

$$
r((k+1) \Delta)=\left\{\begin{array}{rr}
i, \quad & i \in S-\{N\} \text { such that } \sum_{j=1}^{i-1} P_{r(k \Delta), j}(\Delta) \\
& \leq \xi_{k+1}<\sum_{j=1}^{i} P_{r(k \Delta), j}(\Delta), \\
N, & \sum_{j=1}^{N-1} P_{r(k \Delta), j}(\Delta) \leq \xi_{k+1} .
\end{array}\right.
$$

The procedure can be carried out independently to obtain more trajectories.

Define the Euler-Maruyama approximation for (3) by

$$
\begin{aligned}
\mathrm{d} Y(t)= & b(\bar{Y}(t), \bar{Y}(q t), \bar{r}(t)) \mathrm{d} t \\
& +\sigma(\bar{Y}(t), \bar{Y}(q t), \bar{r}(t)) \mathrm{d} W(t), \quad t \in\left[t_{0}, T\right],
\end{aligned}
$$

where $\bar{Y}(t):=Y\left(t_{i}\right), \bar{r}(t):=r\left(t_{i}\right)$ for $t \in\left[t_{i}, t_{i+1}\right), i=0$, $1, \ldots,\left[\log _{\epsilon / q}\left(T / t_{0}\right)\right]$, which

$$
\begin{aligned}
t_{0} & <\frac{t_{0}}{q} \epsilon=t_{1}<\frac{t_{0}}{q^{2}} \epsilon^{2}=t_{2}<\cdots<\frac{t_{0}}{q^{n-1}} \epsilon^{n-1}=t_{n-1} \\
& <\frac{t_{0}}{q^{n}} \epsilon^{n}=t_{n}
\end{aligned}
$$

and $\bar{Y}(\theta)=\xi(\theta), \bar{r}(\theta)=r_{0}, \theta \in\left[q t_{0}, t_{0}\right]$.

By using the method of Lemma 2, we obtain

$$
\begin{aligned}
& \mathbb{E}\left(\sup _{t_{0} \leq t \leq T}|Y(t)|^{p}\right) \leq C, \\
& \mathbb{E}|Y(t)-\bar{Y}(t)|^{p} \leq C \Delta^{p / 2}, \quad t \in\left[t_{0}, T\right],
\end{aligned}
$$

where $\Delta=\max \left\{\Delta_{1}, \Delta_{2}, \ldots, \Delta_{n}\right\}:=(\epsilon / q-1) T, \Delta \rightarrow 0$, when $\epsilon \rightarrow q$.

Lemma 4. If (A1) and (A2) hold, then

$$
\begin{aligned}
& \mathbb{E} \int_{t_{0}}^{T} \mid b(\bar{Y}(s), \bar{Y}(q s), r(s))-\left.b(\bar{Y}(s), \bar{Y}(q s), \bar{r}(s))\right|^{p} \mathrm{~d} s \leq C \Delta, \\
& \mathbb{E} \int_{t_{0}}^{T} \| \sigma(\bar{Y}(s), \bar{Y}(q s), r(s)) \\
&-\sigma(\bar{Y}(s), \bar{Y}(q s), \bar{r}(s)) \|^{p} \mathrm{~d} s \leq C \Delta .
\end{aligned}
$$

Proof. Let $n=\left[\log _{\epsilon / q}\left(T / t_{0}\right)\right]+1$, then

$$
\begin{aligned}
& \mathbb{E} \int_{t_{0}}^{T} \mid b(\bar{Y}(s), \bar{Y}(q s), r(s))-\left.b(\bar{Y}(s), \bar{Y}(q s), \bar{r}(s))\right|^{p} \mathrm{~d} s \\
&=\sum_{i=0}^{n-1} \mathbb{E} \int_{t_{i}}^{t_{i+1}} \mid b(\bar{Y}(s), \bar{Y}(q s), r(s)) \\
&-\left.b\left(\bar{Y}(s), \bar{Y}(q s), r\left(t_{i}\right)\right)\right|^{p} \mathrm{~d} s .
\end{aligned}
$$

By (11), we compute

$$
\begin{aligned}
& \mathbb{E} \int_{t_{i}}^{t_{i+1}} \mid b(\bar{Y}(s), \bar{Y}(q s), r(s)) \\
& -\left.b\left(\bar{Y}(s), \bar{Y}(q s), r\left(t_{i}\right)\right)\right|^{p} \mathrm{~d} s \\
& \leq 2^{p-1} \mathbb{E} \int_{t_{i}}^{t_{i+1}}\left(|b(\bar{Y}(s), \bar{Y}(q s), r(s))|^{p}\right. \\
& \left.+\left|b\left(\bar{Y}(s), \bar{Y}(q s), r\left(t_{i}\right)\right)\right|^{p}\right) \\
& \times I_{\left\{r(s) \neq r\left(t_{i}\right)\right\}} \mathrm{d} s \\
& \leq C \mathbb{E} \int_{t_{i}}^{t_{i+1}}\left(1+|\bar{Y}(s)|^{p}\right. \\
& \left.+|\bar{Y}(q s)|^{p\left(q_{1}+1\right)}\right) I_{\left\{r(s) \neq r\left(t_{i}\right)\right\}} \mathrm{d} s \\
& \leq C \int_{t_{i}}^{t_{i+1}} \mathbb{E}\left[\mathbb { E } \left[\left(1+|\bar{Y}(s)|^{p}\right.\right.\right. \\
& \left.\left.\left.+|\bar{Y}(q s)|^{p\left(q_{1}+1\right)}\right) I_{\left\{r(s) \neq r\left(t_{i}\right)\right\}} \mid r\left(t_{i}\right)\right]\right] \mathrm{d} s \\
& \left.\left.\quad+|\bar{Y}(q s)|^{p\left(q_{1}+1\right)}\right) \mid r\left(t_{i}\right)\right] \\
& \times \int_{t_{i}}^{t_{i+1}} \mathbb{E}\left[\mathbb{E}\left[\left(1+|\bar{Y}(s)|_{\left\{r(s) \neq r\left(t_{i}\right)\right\}}^{p} \mid r\left(t_{i}\right)\right]\right] \mathrm{d} s .\right.
\end{aligned}
$$

By the Markov property, we have

$$
\begin{aligned}
\mathbb{E} & {\left[I_{\left\{r(s) \neq r\left(t_{k}\right)\right\}} \mid r\left(t_{k}\right)\right] } \\
& =\sum_{i \in S} I_{\left\{r\left(t_{k}\right)=i\right\}} P\left(r(s) \neq i \mid r\left(t_{k}\right)=i\right) \\
& =\sum_{i \in S} I_{\left\{r\left(t_{k}\right)=i\right\}} \sum_{j \neq i}\left(\gamma_{i j}\left(s-t_{k}\right)+o\left(s-t_{k}\right)\right) \\
& \leq\left(\max _{1 \leq i \leq n}\left(-\gamma_{i i}\right) \Delta+o(\Delta)\right) \sum_{i \in S} I_{\left\{r\left(t_{k}\right)=i\right\}} \\
& \leq C \Delta .
\end{aligned}
$$


Substituting the above inequality into (29) yields

$$
\begin{aligned}
& \mathbb{E} \int_{t_{i}}^{t_{i+1}}\left|b(\bar{Y}(s), \bar{Y}(q s), r(s))-b\left(\bar{Y}(s), \bar{Y}(q s), r\left(t_{i}\right)\right)\right|^{p} \mathrm{~d} s \\
& \leq C \Delta \int_{t_{i}}^{t_{i+1}}\left[1+\left|\bar{Y}\left(t_{i}\right)\right|^{p}+\left|\bar{Y}\left(q t_{i}\right)\right|^{p\left(q_{1}+1\right)}\right] \mathrm{d} s \\
& \leq C \Delta .
\end{aligned}
$$

So, (28) becomes

$$
\begin{aligned}
& \mathbb{E} \int_{t_{0}}^{T}|b(\bar{Y}(s), \bar{Y}(q s), r(s))-b(\bar{Y}(s), \bar{Y}(q s), \bar{r}(s))|^{p} \mathrm{~d} s \\
& \quad \leq C \Delta .
\end{aligned}
$$

Similarly, we also obtain that

$$
\begin{aligned}
& \mathbb{E} \int_{t_{0}}^{T}\|\sigma(\bar{Y}(s), \bar{Y}(q s), r(s))-\sigma(\bar{Y}(s), \bar{Y}(q s), \bar{r}(s))\|^{p} \mathrm{~d} s \\
& \quad \leq C \Delta .
\end{aligned}
$$

The proof is complete.

Theorem 5. Under (A1) and (A2), for any $p \geq 2$ there exits $C>0$ such that

$$
\mathbb{E}\left(\sup _{t_{0} \leq t \leq T}|X(t)-Y(t)|^{p}\right) \leq C \Delta^{p / 2}
$$

that is, the convergence order of Euler-Maruyama scheme (23) is $1 / 2$.

Proof. Let $\delta>1$ and $\varepsilon>0$; then $\int_{\varepsilon / \delta}^{\varepsilon}(1 / x) \mathrm{d} x=\left.\ln x\right|_{\varepsilon / \delta} ^{\varepsilon}=\ln \delta$, and there is a continuous nonnegative function $\psi_{\delta \varepsilon}(x)(x \geq$ $0)$, which is zero outside $[\varepsilon / \delta, \varepsilon]$, such that

$$
\int_{\varepsilon / \delta}^{\varepsilon} \psi_{\delta \varepsilon}(x) \mathrm{d} x=1, \quad \psi_{\delta \varepsilon}(x) \leq \frac{2}{x \ln \delta}, \quad x>0 .
$$

Define

$$
\begin{gathered}
\phi_{\delta \varepsilon}(x):=\int_{0}^{x} \int_{0}^{y} \psi_{\delta \varepsilon}(z) \mathrm{d} z \mathrm{~d} y, \quad x>0, \\
V_{\delta \varepsilon}(x):=\phi_{\delta \varepsilon}(|x|), \quad x \in \mathbb{R}^{n} .
\end{gathered}
$$

For any $t \in\left[t_{0}, T\right]$, let

$$
\begin{gathered}
Z(t):=X(t)-Y(t), \\
\bar{Z}(t):=Y(t)-\bar{Y}(t), \\
\widetilde{Z}(t):=(X(t), \bar{Y}(t)) \in \mathbb{R}^{2 n} .
\end{gathered}
$$

Using the Itô formula, we have

$$
\begin{gathered}
V_{\delta \varepsilon}(Z(t)) \\
=\int_{t_{0}}^{t}\left\langle\left(V_{\delta \varepsilon}\right)_{x}(Z(s)), b(X(s), X(q s), r(s))\right. \\
-b(\bar{Y}(s), \bar{Y}(q s), \bar{r}(s))\rangle \mathrm{d} s \\
+\frac{1}{2} \int_{t_{0}}^{t} \operatorname{trace}\{(\sigma(X(s), X(q s), r(s)) \\
\quad-\sigma(\bar{Y}(s), \bar{Y}(q s), \bar{r}(s)))^{*} \\
\times\left(V_{\delta \epsilon}\right)_{x x}(Z(s)) \\
\times(\sigma(X(s), X(q s), r(s)) \\
\quad-\sigma(\bar{Y}(s), \bar{Y}(q s), \bar{r}(s)))\} \mathrm{d} s \\
+\int_{t_{0}}^{t}\left\langle\left(V_{\delta \varepsilon}\right)_{x}(Z(s)), \sigma(X(s), X(q s), r(s))\right. \\
-\sigma(\bar{Y}(s), \bar{Y}(q s), \bar{r}(s))\rangle \mathrm{d} W(s) \\
:=I_{1}(t)+I_{2}(t)+I_{3}(t) .
\end{gathered}
$$

By virtue of condition (A1), the Hölder inequality, and Lemma 4, we deduce that

$$
\begin{gathered}
\mathbb{E}\left(\sup _{t_{0} \leq s \leq t}\left|I_{1}(s)\right|^{p}\right) \\
\leq\left(t-t_{0}\right)^{p-1} \mathbb{E} \int_{t_{0}}^{t} \mid\left\langle\left(V_{\delta \varepsilon}\right)_{x}(Z(s)), b(X(s), X(q s), r(s))\right. \\
\quad-b(\bar{Y}(s), \bar{Y}(q s), \bar{r}(s))\rangle\left.\right|^{p} \mathrm{~d} s \\
\leq\left(t-t_{0}\right)^{p-1} \mathbb{E} \int_{t_{0}}^{t} \mid b(X(s), X(q s), r(s)) \\
\leq C \Delta^{p-1} \int_{t_{0}}^{t} \mathbb{E}(\mid b(X(s), X(q s), r(s)) \\
\quad-\left.b(\bar{Y}(s), \bar{Y}(q s), \bar{r}(s))\right|^{p} \mathrm{~d} s \\
\quad+\mid b(\bar{Y}(s), \bar{Y}(q s), r(s)) \\
\left.-\left.b(\bar{Y}(s), \bar{Y}(q s), \bar{r}(s))\right|^{p}\right) \mathrm{d} s \\
\times\left(\mathbb{E}|Z(q s)|^{2 p}\right)^{1 / 2}+\mathbb{E}|\bar{Z}(s)|^{p} \\
\left\{\begin{array}{c}
\int_{t_{0}} \\
\mathbb{E}|Z(s)|^{p}+\left(\mathbb{E} V_{1}^{2 p}(\widetilde{Z}(q s))\right)^{1 / 2}
\end{array}\right.
\end{gathered}
$$




$$
\begin{aligned}
& +\left(\mathbb{E} V_{1}^{2 p}(\widetilde{Z}(q s))\right)^{1 / 2} \\
& \left.\times\left(\mathbb{E}|\bar{Z}(q s)|^{2 p}\right)^{1 / 2}\right\} \mathrm{d} s+C \Delta^{p} .
\end{aligned}
$$

By the Hölder inequality and (A2), we have

$$
\begin{aligned}
& \mathbb{E}\left(\sup _{t_{0} \leq s \leq t}\left|I_{2}(s)\right|^{p}\right) \\
& \leq \frac{1}{2}\left(t-t_{0}\right)^{p-1} \mathbb{E} \\
& \times \int_{t_{0}}^{t} \mid \operatorname{trace}\{(\sigma(X(s), X(q s), r(s)) \\
& -\sigma(\bar{Y}(s), \bar{Y}(q s), \bar{r}(s)))^{*} \\
& \times\left(V_{\delta \varepsilon}\right)_{x x}(Z(s)) \\
& \times(\sigma(X(s), X(q s), r(s)) \\
& -\sigma(\bar{Y}(s), \bar{Y}(q s), \bar{r}(s)))\}\left.\right|^{p} \mathrm{~d} s \\
& \leq C \Delta^{p-1} \int_{t_{0}}^{t} \mathbb{E}\left\{\left\|\left(V_{\delta \epsilon}\right)_{x x}(Z(s))\right\|\right. \\
& \times \| \sigma(X(s), X(q s), r(s)) \\
& \left.-\sigma(\bar{Y}(s), \bar{Y}(q s), \bar{r}(s)) \|^{2}\right\}^{p} \mathrm{~d} s \\
& \leq C \Delta^{p-1} \mathbb{E} \int_{t_{0}}^{t} \frac{1}{|Z(s)|^{p}} \\
& \times\{\| \sigma(X(s), X(q s), r(s)) \\
& -\sigma(\bar{Y}(s), \bar{Y}(q s), r(s)) \|^{2 p} \\
& +\| \sigma(\bar{Y}(s), \bar{Y}(q s), r(s)) \\
& \left.-\sigma(\bar{Y}(s), \bar{Y}(q s), \bar{r}(s)) \|^{2 p}\right\} \\
& \leq C \Delta^{p-1} \int_{t_{0}}^{t}\left\{\mathbb{E}|Z(s)|^{p}\right. \\
& +\frac{1}{\varepsilon^{p}}\left(\mathbb{E} V_{2}^{4 p}(\widetilde{Z}(q s))\right)^{1 / 2}\left(\mathbb{E}|Z(q s)|^{4 p}\right)^{1 / 2} \\
& +\frac{1}{\varepsilon^{p}} \mathbb{E}|\bar{Z}(s)|^{2 p}+\frac{1}{\varepsilon^{p}}\left(\mathbb{E} V_{2}^{4 p}(\widetilde{Z}(q s))\right)^{1 / 2} \\
& \left.\times\left(\mathbb{E}|\bar{Z}(q s)|^{4 p}\right)^{1 / 2}\right\} \mathrm{d} s+\frac{C \Delta^{p}}{\varepsilon^{p}} .
\end{aligned}
$$

Making use of the Burkhold-Davis-Gundy inequality yields

$$
\begin{gathered}
\mathbb{E}\left(\sup _{t_{0} \leq s \leq t}\left|I_{3}(s)\right|^{p}\right) \\
\leq C \Delta^{p / 2-1} \mathbb{E} \int_{t_{0}}^{t} \| \sigma(X(s), X(q s), r(s)) \\
-\sigma(\bar{Y}(s), \bar{Y}(q s), \bar{r}(s)) \|^{p} \mathrm{~d} s \\
\leq C \Delta^{p / 2-1} \mathbb{E} \int_{t_{0}}^{t}(\| \sigma(X(s), X(q s), r(s)) \\
-\sigma(\bar{Y}(s), \bar{Y}(q s), r(s)) \|^{p} \\
+\| \sigma(X(s), X(q s), r(s)) \\
\left.-\sigma(\bar{Y}(s), \bar{Y}(q s), \bar{r}(s)) \|^{p}\right) \mathrm{d} s \\
\leq C \Delta^{p / 2-1} \int_{t_{0}}^{t}\left\{\begin{array}{c}
\mathbb{E}|Z(s)|^{p}+\left(\mathbb{E} V_{2}^{2 p}(\widetilde{Z}(q s))\right)^{1 / 2} \\
\times\left(\mathbb{E}|Z(q s)|^{2 p}\right)^{1 / 2} \\
\times\left(\mathbb{E}|\bar{Z}(s)|^{p}+\left(\left.\mathbb{E}(q s)\right|^{2 p}\right)^{1 / 2}\right\} \mathrm{d} s+C \Delta^{p / 2} .
\end{array}\right.
\end{gathered}
$$

Moreover, by (8), (9), and (25), we have

$$
\begin{gathered}
\mathbb{E} V_{1}^{2 p}(\widetilde{Z}(q s)) \vee \mathbb{E} V_{2}^{4 p}(\widetilde{Z}(q s)) \leq C, \\
\mathbb{E}|\bar{Z}(t)|^{p} \leq C \Delta^{p / 2} .
\end{gathered}
$$

Thus, combing (39), and (40) with (41), for any $t \in\left[t_{0}, T\right]$ and $p \geq 2$, we get

$$
\begin{gathered}
\mathbb{E}\left(\sup _{t_{0} \leq s \leq t}|Z(s)|^{p}\right) \\
\leq 2^{p-1}\left\{\varepsilon^{p}+\mathbb{E}\left(\sup _{t_{0} \leq s \leq t} V_{\delta \varepsilon}^{p}(Z(s))\right)\right\} \\
\leq C\left\{\varepsilon^{p}+\Delta^{3 p / 2-1}+\Delta^{p}+\frac{\Delta^{2 p-1}}{\varepsilon^{p}}+\frac{\Delta^{p}}{\varepsilon^{p}}+\Delta^{p-1}+\Delta^{p / 2}\right. \\
+\Delta^{p / 2-1}\left\{\int_{t_{0}}^{t} \mathbb{E}|Z(s)|^{p} \mathrm{~d} s+\int_{t_{0}}^{t}\left(\mathbb{E}|Z(q s)|^{2 p}\right)^{1 / 2} \mathrm{~d} s\right. \\
\left.\left.+\frac{1}{\varepsilon^{p}} \int_{t_{0}}^{t}\left(\mathbb{E}|Z(q s)|^{4 p}\right)^{1 / 2} \mathrm{~d} s\right\}\right\} .
\end{gathered}
$$


Let $\varepsilon=\Delta^{1 / 2}$, and using the Gronwall inequality, we have

$$
\begin{aligned}
& \mathbb{E}\left(\sup _{t_{0} \leq s \leq t}|Z(s)|^{p}\right) \\
& \leq C\left\{\Delta^{p / 2}+\Delta^{(p / 2)-1} \int_{t_{0}}^{t}\left(\mathbb{E}|Z(q s)|^{2 p}\right)^{1 / 2} \mathrm{~d} s\right. \\
&\left.+\Delta^{-1} \int_{t_{0}}^{t}\left(\mathbb{E}|Z(q s)|^{4 p}\right)^{1 / 2} \mathrm{~d} s\right\} .
\end{aligned}
$$

Let

$$
\begin{array}{r}
p_{i}:=\left(\left[\log _{\epsilon / q} \frac{T}{t_{0}}\right]+2-i\right) p 4^{\left[\log _{\epsilon / q}\left(T / t_{0}\right)\right]+1-i}, \\
i=1,2, \ldots,\left[\log _{\epsilon / q} \frac{T}{t_{0}}\right]+1
\end{array}
$$

by $p \geq 2$, it is easy to see that $p_{i} \geq 2$ such that

$$
\begin{array}{r}
4 p_{i+1}<p_{i}, \quad p_{\left[\log _{\epsilon / q}\left(T / t_{0}\right)\right]+1}=p, \\
i=1,2, \ldots,\left[\log _{\epsilon / q} \frac{T}{t_{0}}\right] .
\end{array}
$$

Noting that $Z(s)=0$ for $s \in\left[q t_{0}, t_{0}\right]$ and substituting $\epsilon<1$ into (44) yields that

$$
\begin{gathered}
\mathbb{E}\left(\sup _{t_{0} \leq s \leq\left(t_{0} / q\right) \epsilon}|Z(s)|^{p_{1}}\right) \\
\leq C\left\{\Delta^{p_{1} / 2}+\Delta^{p_{1} / 2-1} \int_{t_{0}}^{\left(t_{0} / q\right) \epsilon}\left(\mathbb{E}|Z(q s)|^{2 p_{1}}\right)^{1 / 2} \mathrm{~d} s\right. \\
\left.+\Delta^{-1} \int_{t_{0}}^{\left(t_{0} / q\right) \epsilon}\left(\mathbb{E}|Z(q s)|^{4 p_{1}}\right)^{1 / 2} \mathrm{~d} s\right\} \\
\leq C \\
\left\{\Delta^{p_{1} / 2}+\Delta^{p_{1} / 2-1} \int_{q t_{0}}^{t_{0}}\left(\mathbb{E}|Z(s)|^{2 p_{1}}\right)^{1 / 2} \mathrm{~d} s\right. \\
\left.+\Delta^{-1} \int_{q t_{0}}^{t_{0}}\left(\mathbb{E}|Z(s)|^{4 p_{1}}\right)^{1 / 2} \mathrm{~d} s\right\} \\
\leq C \Delta^{p_{1} / 2} .
\end{gathered}
$$

Using (46) and the Hölder inequality, further gives that

$$
\begin{gathered}
\mathbb{E}\left(\sup _{t_{0} \leq s \leq\left(t_{0} / q^{2}\right) \epsilon^{2}}|Z(s)|^{p_{2}}\right) \\
\leq C\left\{\Delta^{p_{2} / 2}+\Delta^{p_{2} / 2-1} \int_{t_{0}}^{\left(t_{0} / q^{2}\right) \epsilon^{2}}\left(\mathbb{E}|Z(q s)|^{2 p_{2}}\right)^{1 / 2} \mathrm{~d} s\right. \\
\left.+\Delta^{-1} \int_{t_{0}}^{\left(t_{0} / q^{2}\right) \epsilon^{2}}\left(\mathbb{E}|Z(q s)|^{4 p_{2}}\right)^{1 / 2} \mathrm{~d} s\right\}
\end{gathered}
$$

$$
\begin{aligned}
& \leq C\left\{\Delta^{p_{2} / 2}+\Delta^{p_{2} / 2-1} \int_{t_{0}}^{\left(t_{0} / q\right) \epsilon}\left(\mathbb{E}|Z(s)|^{p_{1}}\right)^{p_{2} / p_{1}} \mathrm{~d} s\right. \\
& \left.+\Delta^{-1} \int_{t_{0}}^{\left(t_{0} / q\right) \epsilon}\left(\mathbb{E}|Z(s)|^{p_{1}}\right)^{2 p_{2} / p_{1}} \mathrm{~d} s\right\} \\
& \leq C \Delta^{p_{2} / 2} .
\end{aligned}
$$

Repeating the previous procedures, the desired result follows.

In this section, under general conditions, we reveal that the convergence order of Euler-Maruyama scheme for stochastic pantograph equations with Markovian switching and Brownian motion is $1 / 2$. In Section 3, we will discuss the convergence rate for stochastic pantograph equation with Markovian switching and pure jumps.

\section{Convergence Rate for Markovian Switching and Pure Jumps Case}

Let $\mathscr{B}(\mathbb{R})$ be the Borel $\sigma$-algebra on $\mathbb{R}$, and $\lambda(\mathrm{d} x)$ a $\sigma$ finite measure defined on $\mathscr{B}(\mathbb{R})$. Let $p=(p(t)), t \in$ $D_{p}$, be a stationary $\mathscr{F}_{t}$-Poisson point process on $\mathbb{R}$ with characteristic measure $\lambda(\cdot)$. Denote by $N(\mathrm{~d} t, \mathrm{~d} u)$ the Poisson counting measure associated with $p$, that is, $N(t, U)=$ $\sum_{s \in D_{p}, s \leq t} I_{U}(p(s))$ for $U \in \mathscr{B}(\mathbb{R})$. Let $\widetilde{N}(\mathrm{~d} t, \mathrm{~d} u):=N(\mathrm{~d} t, \mathrm{~d} u)-$ $\mathrm{d} t \lambda(\mathrm{d} u)$ be the compensated Poisson measure associated with $N(\mathrm{~d} t, \mathrm{~d} u)$. In what follows, we further assume that $\int_{U}|u|^{p} \lambda(u)<\infty$ for any $p \geq 2$.

In this section, we consider the following stochastic pantograph equation with Markovian switching and pure jumps on $\mathbb{R}^{n}$ :

$$
\begin{aligned}
\mathrm{d} X(t)= & b(X(t), X(q t), r(t)) \mathrm{d} t \\
& +\int_{U} h(X(t), X(q t), u) \widetilde{N}(\mathrm{~d} t, \mathrm{~d} u), \quad t \in\left[t_{0}, T\right]
\end{aligned}
$$

with initial data $X(\theta)=\xi(\theta)$ and $r(\theta)=r_{0}, \theta \in\left[q t_{0}, t_{0}\right]$.

We assume that

(A1) $b: \mathbb{R}^{n} \times \mathbb{R}^{n} \times S \rightarrow \mathbb{R}^{n}$ and there exists $L_{1}>0$ such that

$$
\begin{aligned}
& \left|b\left(x_{1}, y_{1}, j\right)-b\left(x_{2}, y_{2}, j\right)\right| \\
& \quad \leq L_{1}\left|x_{1}-x_{2}\right|+V_{1}\left(y_{1}, y_{2}\right)\left|y_{1}-y_{2}\right|
\end{aligned}
$$

for $x_{i}, y_{i} \in \mathbb{R}^{n}, i=1,2, j \in S$;

(A3) $h: \mathbb{R}^{n} \times \mathbb{R}^{n} \times U \rightarrow \mathbb{R}^{n}$ and there exists $L_{3}>0$ such that

$$
\begin{aligned}
& \left|h\left(x_{1}, y_{1}, u\right)-h\left(x_{2}, y_{2}, u\right)\right| \\
& \quad \leq\left(L_{3}\left|x_{1}-x_{2}\right|+V_{3}\left(y_{1}, y_{2}\right)\left|y_{1}-y_{2}\right|\right)|u|
\end{aligned}
$$


for $x_{i}, y_{i} \in \mathbb{R}^{n}, i=1,2$, and $u \in U$, where $V_{3}: \mathbb{R}^{n} \times$ $\mathbb{R}^{n} \rightarrow \mathbb{R}_{+}$such that

$$
V_{3}(x, y) \leq K_{3}\left(1+|x|^{q_{3}}+|y|^{q_{3}}\right)
$$

for some $K_{3}>0, q_{3} \geq 1$ and arbitrary $x, y \in \mathbb{R}^{n}$.

From (A3), the jump coefficient may be also highly nonlinear. We define the Euler-Maruyama scheme associated with (49) by

$$
\begin{aligned}
\mathrm{d} Y(t)= & b(\bar{Y}(t), \bar{Y}(q t), \bar{r}(t)) \mathrm{d} t \\
& +\int_{U} h(\bar{Y}(t), \bar{Y}(q t), u) \widetilde{N}(\mathrm{~d} t, \mathrm{~d} u),
\end{aligned}
$$

where $\bar{Y}(t):=Y\left(t_{i}\right), \bar{r}(t):=r\left(t_{i}\right)$ for $t \in\left[t_{i}, t_{i+1}\right), i=$ $0,1, \ldots, n-1$, and $\bar{Y}(\theta)=\xi(\theta), \bar{r}(\theta)=r_{0}$, for $\theta \in\left[q t_{0}, t_{0}\right]$.

In order to state the main theorem, the following two lemmas are useful.

Lemma 6 (see [16]). Let $\Phi: \mathbb{R}_{+} \times U \rightarrow \mathbb{R}^{n}$ and assume that

$$
\int_{t_{0}}^{t} \int_{U} \mathbb{E}|\Phi(s, u)|^{p} \lambda(\mathrm{d} u) \mathrm{d} s<\infty, \quad t_{0}>0, \quad p \geq 2 .
$$

Then there exists $D(p)>0$ such that

$$
\begin{aligned}
\mathbb{E}\left(\sup _{t_{0} \leq s \leq t}\left|\int_{t_{0}}^{s} \int_{U} \Phi(r, u) \widetilde{N}(\mathrm{~d} u, \mathrm{~d} s)\right|^{p}\right) \\
\leq D(p)\left\{\mathbb{E}\left(\int_{t_{0}}^{t} \int_{U}|\Phi(s, u)|^{2} \lambda(\mathrm{d} u) \mathrm{d} s\right)^{p / 2}\right. \\
\left.+\mathbb{E} \int_{t_{0}}^{t} \int_{U}|\Phi(s, u)|^{p} \lambda(\mathrm{d} u) \mathrm{d} s\right\} .
\end{aligned}
$$

Lemma 7. Let (A1) and (A3) hold. Then (49) has a unique global solution $(X(t))_{t \in\left[t_{0}, T\right]}$. Moreover, for any $p \geq 2$ there exists $C>0$ such that

$$
\begin{gathered}
\mathbb{E}\left(\sup _{t_{0} \leq t \leq T}|X(t)|^{p}\right) \vee \mathbb{E}\left(\sup _{t_{0} \leq t \leq T}|Y(t)|^{p}\right) \leq C, \\
\mathbb{E}|Y(t)-\bar{Y}(t)|^{p} \leq C \Delta .
\end{gathered}
$$

Proof. The proof is very similar to that of Lemma 2 and (25).

Now we present the main theorem in this section.

Theorem 8. Let $(A 1)$ and $(A 3)$ hold. For any $p \geq 2$ and arbitrary $\theta, \alpha \in(0,1)$, there exists $C>0$, independent of $\Delta$, such that

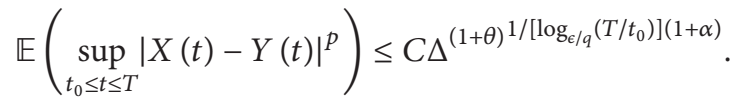

Proof. The proof is similar to that of Theorem 5. Set

$$
\begin{gathered}
Z(t):=X(t)-Y(t), \\
\bar{Z}(t):=Y(t)-\bar{Y}(t), \\
\widetilde{Z}(t):=(X(t), \bar{Y}(t)) \in \mathbb{R}^{2 n}, \quad t \in\left[t_{0}, T\right] .
\end{gathered}
$$

Define

$$
\begin{gathered}
\Gamma_{1}(t):=b(X(t), X(q t), r(t))-b(\bar{Y}(t), \bar{Y}(q t), \bar{r}(t)), \\
\Gamma_{2}(t, u):=h(X(t), X(q t), u)-h(\bar{Y}(t), \bar{Y}(q t), u) .
\end{gathered}
$$

Using the Itô formula and the Taylor expansion we have that for $t \in\left[t_{0}, T\right]$

$$
\begin{aligned}
& V_{\delta \varepsilon}(Z(t))=\int_{t_{0}}^{t}\left\langle\left(V_{\delta \varepsilon}\right)_{x}(Z(s)), \Gamma_{1}(s)\right\rangle \mathrm{d} s \\
& +\int_{t_{0}}^{t} \int_{U}\left\{V_{\delta \varepsilon}\left(Z(s)+\Gamma_{2}(s, u)\right)\right. \\
& -V_{\delta \varepsilon}(Z(s)) \\
& \left.-\left\langle\left(V_{\delta \varepsilon}\right)_{x}(Z(s)), \Gamma_{2}(s, u)\right\rangle\right\} \\
& \times \lambda(\mathrm{d} u) \mathrm{d} s \\
& +\int_{t_{0}}^{t} \int_{U}\left\{V_{\delta \varepsilon}\left(Z(s)+\Gamma_{2}(s, u)\right)\right. \\
& \left.-V_{\delta \varepsilon}(Z(s))\right\} \widetilde{N}(\mathrm{~d} u, \mathrm{~d} s) \\
& =\int_{t_{0}}^{t}\left\langle\left(V_{\delta \varepsilon}\right)_{x}(Z(s)), \Gamma_{1}(s)\right\rangle \mathrm{d} s \\
& +\int_{t_{0}}^{t} \int_{U}\left\{\int _ { 0 } ^ { 1 } \left\langle\left(V_{\delta \varepsilon}\right)_{x}\left(Z(s)+\theta \Gamma_{2}(s, u)\right)\right.\right. \\
& \left.\left.-\left(V_{\delta \varepsilon}\right)_{x}(Z(s)), \Gamma_{2}(s, u)\right\rangle d \theta\right\} \\
& \times \lambda(\mathrm{d} u) \mathrm{d} s \\
& +\int_{t_{0}}^{t} \int_{U}\left\{\int _ { 0 } ^ { 1 } \left\langle\left(V_{\delta \varepsilon}\right)_{x}\left(Z(s)+\theta \Gamma_{2}(s, u)\right),\right.\right. \\
& \left.\left.\Gamma_{2}(s, u)\right\rangle \mathrm{d} \theta\right\} \widetilde{N}(\mathrm{~d} u, \mathrm{~d} s) .
\end{aligned}
$$

By the property of $V_{\delta \varepsilon}(x)$, we deduce that

$$
\begin{aligned}
|Z(t)| & \leq \varepsilon+V_{\delta \varepsilon}(Z(t)) \\
& \leq \varepsilon+\int_{t_{0}}^{t}\left|\Gamma_{1}(s)\right| \mathrm{d} s+2 \int_{t_{0}}^{t} \int_{U}\left|\Gamma_{2}(s, u)\right| \lambda(\mathrm{d} u) \mathrm{d} s
\end{aligned}
$$




$$
\begin{array}{r}
+\int_{t_{0}}^{t} \int_{U}\left\{\int _ { 0 } ^ { 1 } \left\langle\left(V_{\delta \varepsilon}\right)_{x}\left(Z(s)+\theta \Gamma_{2}(s, u)\right),\right.\right. \\
\left.\left.\Gamma_{2}(s, u)\right\rangle \mathrm{d} \theta\right\} \widetilde{N}(\mathrm{~d} u, \mathrm{~d} s), \\
t \in\left[t_{0}, T\right] .
\end{array}
$$

From (8), (52), and (56), we compute that for any $p \geq 2$

$$
\mathbb{E}\left(\sup _{t_{0} \leq t \leq T} V_{1}^{p}(\widetilde{Z}(q s))\right) \vee \mathbb{E}\left(\sup _{t_{0} \leq t \leq T} V_{3}^{p}(\widetilde{Z}(q s))\right) \leq C .
$$

Applying Lemma 6, Lemma 4, (57), and the Hölder inequality, we obtain that

$$
\begin{aligned}
& \mathbb{E}\left(\sup _{t_{0} \leq s \leq t}|Z(s)|^{p}\right) \\
& \leq 2^{p-1}\left\{\varepsilon^{p}+\mathbb{E}\left(\sup _{t_{0} \leq s \leq t} V_{\delta \varepsilon}^{p}(Z(s))\right)\right\} \\
& \leq C\left\{\varepsilon^{p}+\int_{t_{0}}^{t} \mathbb{E}\left|\Gamma_{1}(s)\right|^{p} \mathrm{~d} s\right. \\
& +\int_{t_{0}}^{t} \int_{U} \mathbb{E}\left|\Gamma_{2}(s, u)\right|^{p} \lambda(\mathrm{d} u) \mathrm{d} s \\
& \left.+\mathbb{E}\left(\int_{t_{0}}^{t} \int_{U}\left|\Gamma_{2}(s, u)\right|^{2} \lambda(\mathrm{d} u) \mathrm{d} s\right)^{p / 2}\right\} \\
& \leq C\left\{\varepsilon^{p}+\int_{t_{0}}^{t} \mathbb{E}\left|\Gamma_{1}(s)\right|^{p} \mathrm{~d} s\right. \\
& \left.+\int_{t_{0}}^{t} \int_{U} \mathbb{E}\left|\Gamma_{2}(s, u)\right|^{p} \lambda(\mathrm{d} u) \mathrm{d} s\right\} \\
& \leq C\left\{\varepsilon^{p}+\int_{t_{0}}^{t} \mathbb{E}(|X(s)-\bar{Y}(s)|\right. \\
& \left.+V_{1}(\widetilde{Z}(q s))|X(q s)-\bar{Y}(q s)|\right)^{p} \mathrm{~d} s \\
& +\int_{t_{0}}^{t} \mathbb{E} \mid b(\bar{Y}(s), \bar{Y}(q s), r(s)) \\
& -\left.b(\bar{Y}(s), \bar{Y}(q s), \bar{r}(s))\right|^{p} \mathrm{~d} s \\
& +\int_{t_{0}}^{t} \mathbb{E}(|X(s)-\bar{Y}(s)| \\
& \left.\left.+V_{3}(\widetilde{Z}(q s))|X(q s)-\widetilde{Y}(q s)|\right)^{p} \mathrm{~d} s\right\} \\
& \leq C\left\{\varepsilon^{p}+\Delta+\int_{t_{0}}^{t}\left\{\mathbb{E}|Z(s)|^{p}\right.\right. \\
& +\mathbb{E}\left(V_{1}^{p}(\widetilde{Z}(q s))|Z(q s)|^{p}\right) \\
& +\mathbb{E}\left(V_{1}^{p}(\widetilde{Z}(q s))|\bar{Z}(q s)|^{p}\right)
\end{aligned}
$$

$$
\begin{gathered}
+\mathbb{E}\left(V_{3}^{p}(\widetilde{Z}(q s))|Z(q s)|^{p}\right) \\
\left.\left.+\mathbb{E}\left(V_{3}^{p}(\widetilde{Z}(q s))|\bar{Z}(q s)|^{p}\right)\right\} \mathrm{d} s\right\} \\
\leq C\left\{\varepsilon^{p}+\Delta+\int_{t_{0}}^{t} \mathbb{E}|Z(s)|^{p} \mathrm{~d} s+\int_{t_{0}}^{t} \mathbb{E}|Z(q s)|^{p} \mathrm{~d} s\right\} .
\end{gathered}
$$

Together with the Gronwall inequality and taking $\varepsilon=$ $\Delta^{1 / p}$, we get

$$
\mathbb{E}\left(\sup _{t_{0} \leq s \leq t}|Z(s)|^{p}\right) \leq C\left\{\Delta+\int_{t_{0}}^{t} \mathbb{E}|Z(q s)|^{p} \mathrm{~d} s\right\}
$$

For $\theta \in(0,1)$ and any $\alpha \in(0,1)$, let

$$
\begin{array}{r}
p_{i}:=p(1+\theta)^{\left(\left[\log _{\epsilon / q}\left(T / t_{0}\right)\right]+1-i\right)(1+\alpha)}, \\
i=1,2, \ldots,\left[\log _{\epsilon / q} \frac{T}{t_{0}}\right]+1 .
\end{array}
$$

It is easy to see that

$$
\begin{array}{r}
(1+\theta) p_{i+1}<p_{i}, \quad p_{\left[\log _{\epsilon / q}\left(T / t_{0}\right)\right]+1}=p \\
i=1,2, \ldots,\left[\log _{\epsilon / q} \frac{T}{t_{0}}\right]
\end{array}
$$

Noting that $Z(t)=\bar{Z}(t)=0$ for $t \in\left[q t_{0}, t_{0}\right]$, from (65) we obtain

$$
\mathbb{E}\left(\sup _{t_{0} \leq s \leq\left(t_{0} / q\right) \epsilon}|Z(s)|^{p_{1}}\right) \leq C\left\{\Delta+\int_{q t_{0}}^{t_{0}} \mathbb{E}|Z(s)|^{p_{1}} \mathrm{~d} s\right\} \leq C \Delta .
$$

Then, together with (67) and the Hölder inequality, it further gives that

$$
\begin{aligned}
& \mathbb{E}\left(\sup _{t_{0} \leq s \leq\left(t_{0} / q^{2}\right) \epsilon^{2}}|Z(s)|^{p_{2}}\right) \\
& \leq C\left\{\Delta+\int_{t_{0}}^{\left(t_{0} / q\right) \epsilon}\left(\mathbb{E}|Z(s)|^{p_{2}(1+\theta)}\right)^{1 /(1+\theta)} \mathrm{d} s\right\} \\
& \leq C\left\{\Delta+\int_{t_{0}}^{\left(t_{0} / q\right) \varepsilon}\left(\mathbb{E}|Z(s)|^{p_{1}}\right)^{p_{2} / p_{1}} \mathrm{~d} s\right\} \\
& \leq C \Delta^{p_{2} / p_{1}} .
\end{aligned}
$$


Similarly,

$$
\begin{aligned}
& \mathbb{E}\left(\sup _{t_{0} \leq s \leq\left(t_{0} / q^{3}\right) \epsilon^{3}}|Z(s)|^{p_{3}}\right) \\
& \leq C\left\{\Delta+\int_{t_{0}}^{\left(t_{0} / q^{2}\right) \epsilon^{2}}\left(\mathbb{E}|Z(s)|^{p_{3}(1+\theta)}\right)^{1 /(1+\theta)} \mathrm{d} s\right\} \\
& \leq C\left\{\Delta+\int_{t_{0}}^{\left(t_{0} / q^{2}\right) \epsilon^{2}}\left(\mathbb{E}|Z(s)|^{p_{2}}\right)^{p_{3} / p_{2}} \mathrm{~d} s\right\} \\
& \leq C \Delta^{p_{3} / p_{1}} .
\end{aligned}
$$

Repeating the previous procedures, we have

$$
\mathbb{E}\left(\sup _{t_{0} \leq s \leq T}|Z(s)|^{p}\right) \leq C \Delta^{(1+\theta)^{1 / \log _{\epsilon} / q^{\left.\left(T / t_{0}\right)\right](1+\alpha)}} .}
$$

The proof is complete.

\section{Acknowledgments}

This work was supported by the National Natural Science Foundation of China (nos. 61104062 and 61174077), Jiangsu Qing Lan Project, and PAPD.

\section{References}

[1] H. Dong, Z. Wang, D. W. C. Ho, and H. Gao, "Robust $H_{\infty}$ filtering for Markovian jump systems with randomly occurring nonlinearities and sensor saturation: the finite-horizon case," IEEE Transactions on Signal Processing, vol. 59, no. 7, pp. 30483057, 2011.

[2] X. Mao, Stochastic Differential Equations and Applications, Horwood, Chichester, UK, Second edition, 2008.

[3] X. Mao and C. Yuan, Stochastic Differential Equations with Markovian Switching, Imperial College Press, London, UK, 2006.

[4] Y. Shen and J. Wang, "An improved algebraic criterion for global exponential stability of recurrent neural networks with timevarying delays," IEEE Transactions on Neural Networks, vol. 19, no. 3, pp. 528-531, 2008.

[5] Z. Wang, Y. Liu, and X. Liu, "Exponential stabilization of a class of stochastic system with Markovian jump parameters and mode-dependent mixed time-delays," IEEE Transactions on Automatic Control, vol. 55, no. 7, pp. 1656-1662, 2010.

[6] J. Bao, B. Böttcher, X. Mao, and C. Yuan, "Convergence rate of numerical solutions to SFDEs with jumps," Journal of Computational and Applied Mathematics, vol. 236, no. 2, pp. 119-131, 2011.

[7] L. Ronghua and C. Zhaoguang, "Convergence of numerical solution to stochastic delay differential equation with Poisson jump and Markovian switching," Applied Mathematics and Computation, vol. 184, no. 2, pp. 451-463, 2007.

[8] I. Gyöngy and M. Rásonyi, "A note on Euler approximations for SDEs with Hölder continuous diffusion coefficients," Stochastic Processes and their Applications, vol. 121, no. 10, pp. 2189-2200, 2011.
[9] X. Mao, C. Yuan, and G. Yin, "Approximations of EulerMaruyama type for stochastic differential equations with Markovian switching, under non-Lipschitz conditions," Journal of Computational and Applied Mathematics, vol. 205, no. 2, pp. 936-948, 2007.

[10] F. Wu, X. Mao, and K. Chen, “The Cox-Ingersoll-Ross model with delay and strong convergence of its Euler-Maruyama approximate solutions," Applied Numerical Mathematics, vol. 59, no. 10, pp. 2641-2658, 2009.

[11] J. Bao and C. Yuan, "Convergence rate of EM scheme for SDDEs," http://arxiv.org/abs/1111.4130.

[12] Z. Fan, M. Liu, and W. Cao, "Existence and uniqueness of the solutions and convergence of semi-implicit Euler methods for stochastic pantograph equations," Journal of Mathematical Analysis and Applications, vol. 325, no. 2, pp. 1142-1159, 2007.

[13] Z. Fan, M. Song, and M. Liu, "The $\alpha$ th moment stability for the stochastic pantograph equation," Journal of Computational and Applied Mathematics, vol. 233, no. 2, pp. 109-120, 2009.

[14] L. Ronghua, L. Min, and P. Wan-kai, "Convergence of numerical solutions to stochastic pantograph equations with Markovian switching," Applied Mathematics and Computation, vol. 215, no. 1, pp. 414-422, 2009.

[15] Y. Xiao and H. Y. Zhang, "A note on convergence of semiimplicit Euler methods for stochastic pantograph equations," Computers \& Mathematics with Applications, vol. 59, no. 4, pp. 1419-1424, 2010.

[16] C. Marinelli, C. Prévôt, and M. Röckner, "Regular dependence on initial data for stochastic evolution equations with multiplicative Poisson noise," Journal of Functional Analysis, vol. 258, no. 2, pp. 616-649, 2010. 


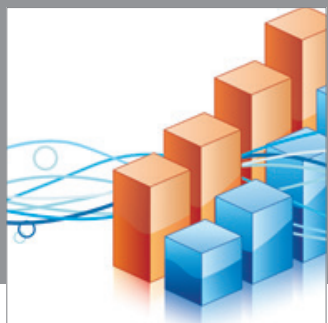

Advances in

Operations Research

mansans

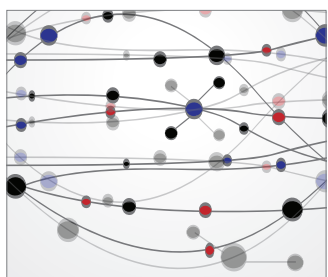

The Scientific World Journal
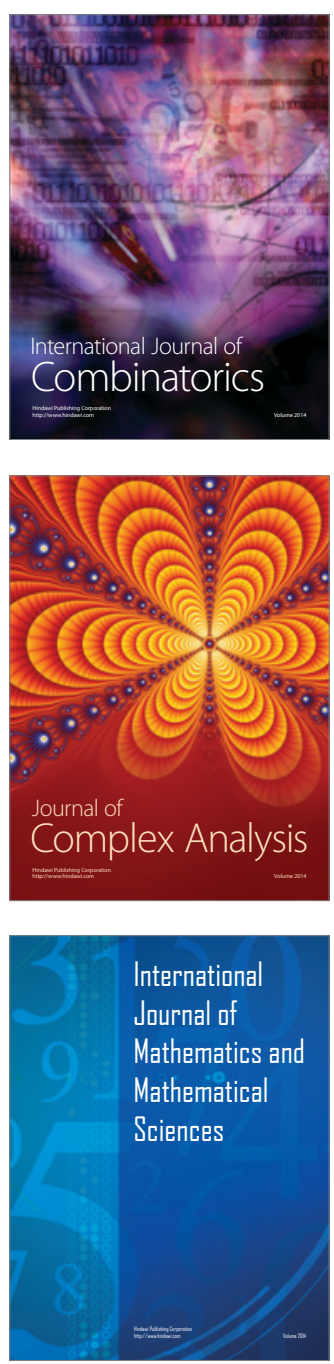
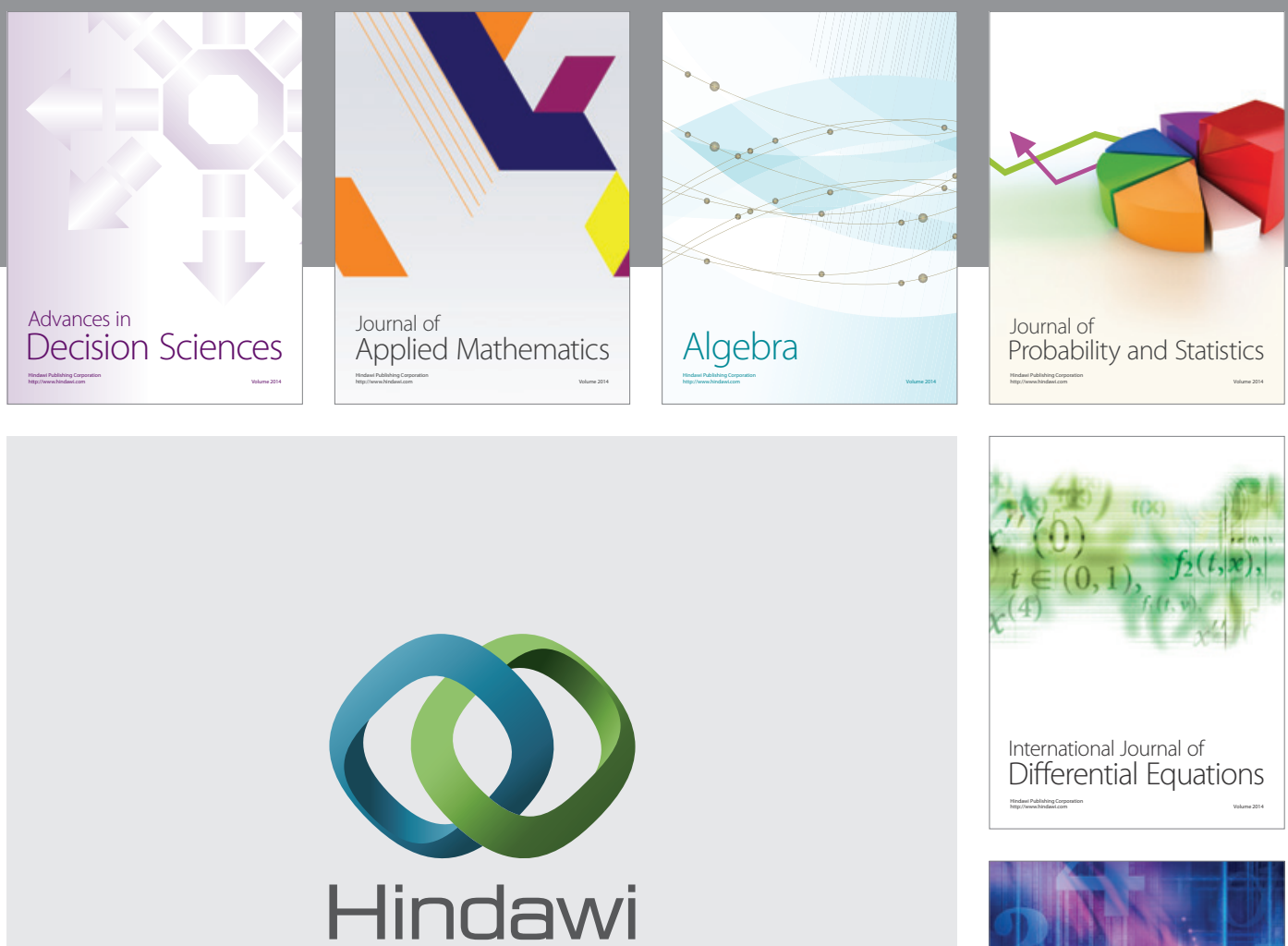

Submit your manuscripts at http://www.hindawi.com
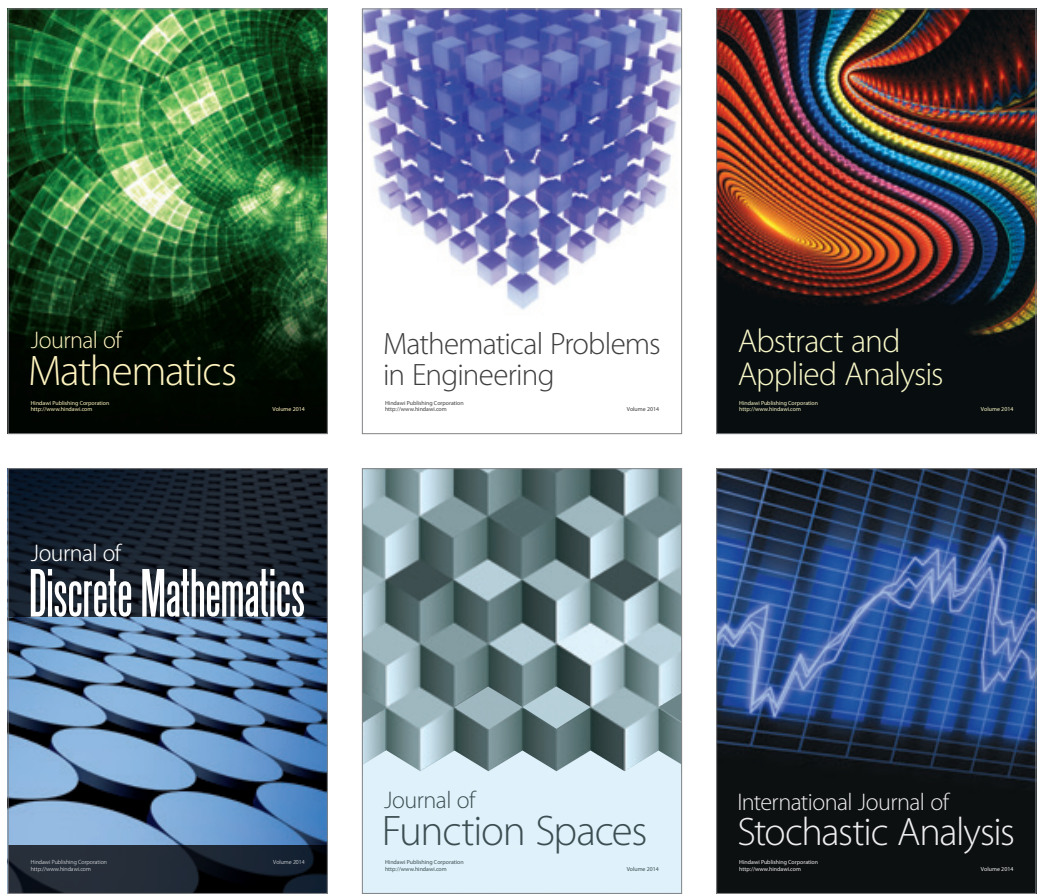

Journal of

Function Spaces

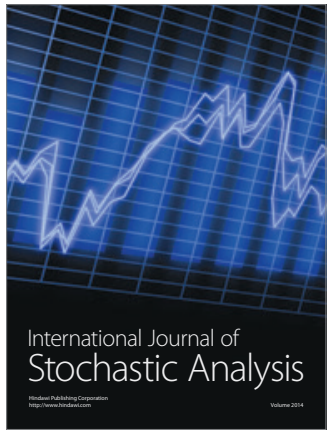

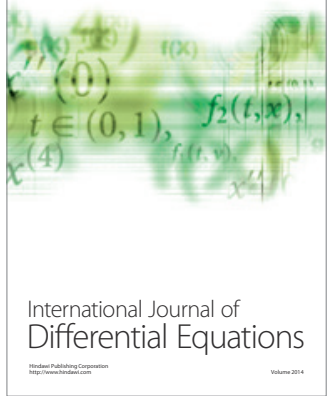
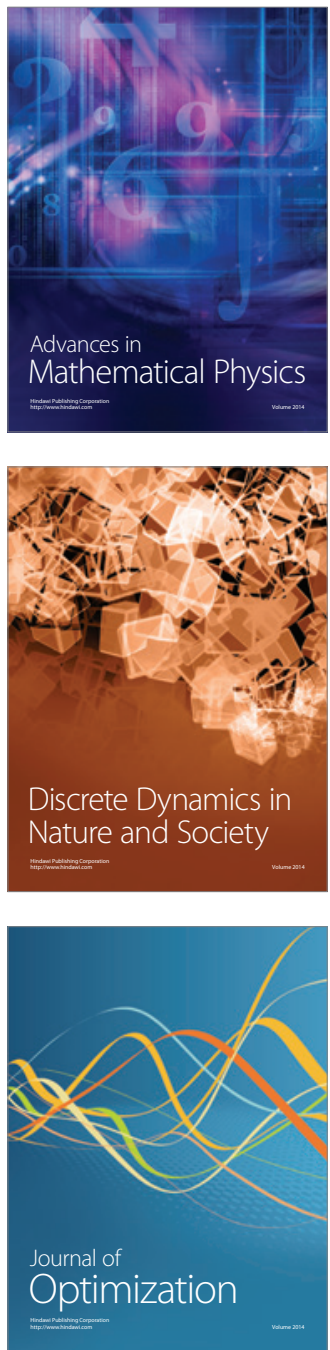